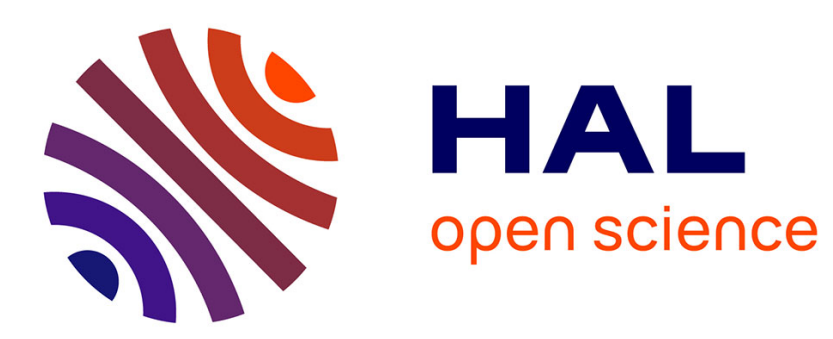

\title{
Hot strip method: application to thermal characterisation of orthotropic media
}

\author{
Claire Gobbé, Sébastien Iserna, Bruno Ladevie
}

\section{To cite this version:}

Claire Gobbé, Sébastien Iserna, Bruno Ladevie. Hot strip method: application to thermal characterisation of orthotropic media. International Journal of Thermal Sciences, 2004, 43 (10), pp.951 - 958. 10.1016/j.ijthermalsci.2004.02.002 . hal-01651374

\section{HAL Id: hal-01651374 https://imt-mines-albi.hal.science/hal-01651374}

Submitted on 7 Nov 2019

HAL is a multi-disciplinary open access archive for the deposit and dissemination of scientific research documents, whether they are published or not. The documents may come from teaching and research institutions in France or abroad, or from public or private research centers.
L'archive ouverte pluridisciplinaire HAL, est destinée au dépôt et à la diffusion de documents scientifiques de niveau recherche, publiés ou non, émanant des établissements d'enseignement et de recherche français ou étrangers, des laboratoires publics ou privés. 


\title{
Hot strip method: application to thermal characterisation of orthotropic media
}

\author{
Claire Gobbé ${ }^{\mathrm{a}, *}$, Sébastien Iserna ${ }^{\mathrm{a}}$, Bruno Ladevie $^{\mathrm{b}}$ \\ a TREFLE (UMR 8508), esplanade des Arts et Métiers, 33405 Talence cedex, France \\ b École des mines d'Albi Carmaux, centre énergétique et environnement, campus Jarlard, 81013 Albi CT cedex 09, France
}

\begin{abstract}
This study deals with determination of the thermal conductivity tensor for orthotropic media and more specifically for multilayers with isotropic thermal characteristics in the planes parallel to the layers. The work described has been conducted using two ordinary experimental devices: a device based on the hot-wire method and a device based on the hot-strip method. Hot-wire measurements give the thermal conductivity in the planes parallel to layers. Introducing this value in a model adequate to describe orthotropic behaviour and using an appropriate identification method, hot-strip measurements then give the transverse thermal conductivity. The validity of this approach is demonstrated by the results obtained on a stratified medium with known thermal characteristics. Then, transverse thermal measurements were made on a non-woven wood fibre insulator.
\end{abstract}

Keywords: Hot wire; Hot strip; Thermal conductivity; Orthotropic media; Multilayer; Quadrupole modelling; Parameter estimation

\section{Introduction}

In many industries (aviation, wood, textile, etc.), the materials used have a highly anisotropic structure, making it difficult to measure the thermal properties of these materials using conventional measuring instruments (hot-wire and hot-strip, flash method, guarded hot plate, etc.), since most such instruments, as normally used, give an apparent total conductivity of the material.

This being the case, certain measurement methods were modified by adapting the models and associated identification procedures to obtain thermal conductivity or diffusivity values in the main directions of diffusion. This can be illustrated by the flash method, where partial irradiation of the front and installation of two thermocouples at the rear allowed identification of a radial diffusivity that was different from the axial diffusivity [1]. The accuracy of the results obtained strongly depends on how accurately the thermocouples are positioned. One way of solving this problem is by the use of infrared thermography for the acquisition of a

\footnotetext{
* Corresponding author.

E-mail addresses: gobbe@bordeaux.ensam.fr (C. Gobbé), ladevie@enstimac.fr (B. Ladevie).
}

larger number of temperature measurements. This technique allows measurement of the longitudinal diffusivities, $a_{x}$ and $a_{y}$, on a thin plate [2].

The hot-wire method [3-8] and hot-strip method [914] allow direct identification of the thermal conductivity of a material assumed to be homogeneous and isotropic. The simplest devices are those for which the temperature is measured on the heating element itself.

Few changes have been proposed to adapt this type of device for characterisation of anisotropic materials. A recent study concerns determination of the conductivity of the strata forming a dual layer, where the strata are perpendicular to the wire axis [15]. In this application, the stated aim led to instrumentation that was more complex than conventional devices because of the necessity to place a thermocouple in the location of each stratum.

Our approach was different since the aim was to use associated two measuring devices in their simplest technological version and to determine the components of the conductivity tensor of an orthotropic material by adapting the identification procedures.

Actually, because of the geometry of the hot-wire heating element (very long cylindrical wire), the dissipated heat flux diffuses into the medium perpendicular to the wire provided 


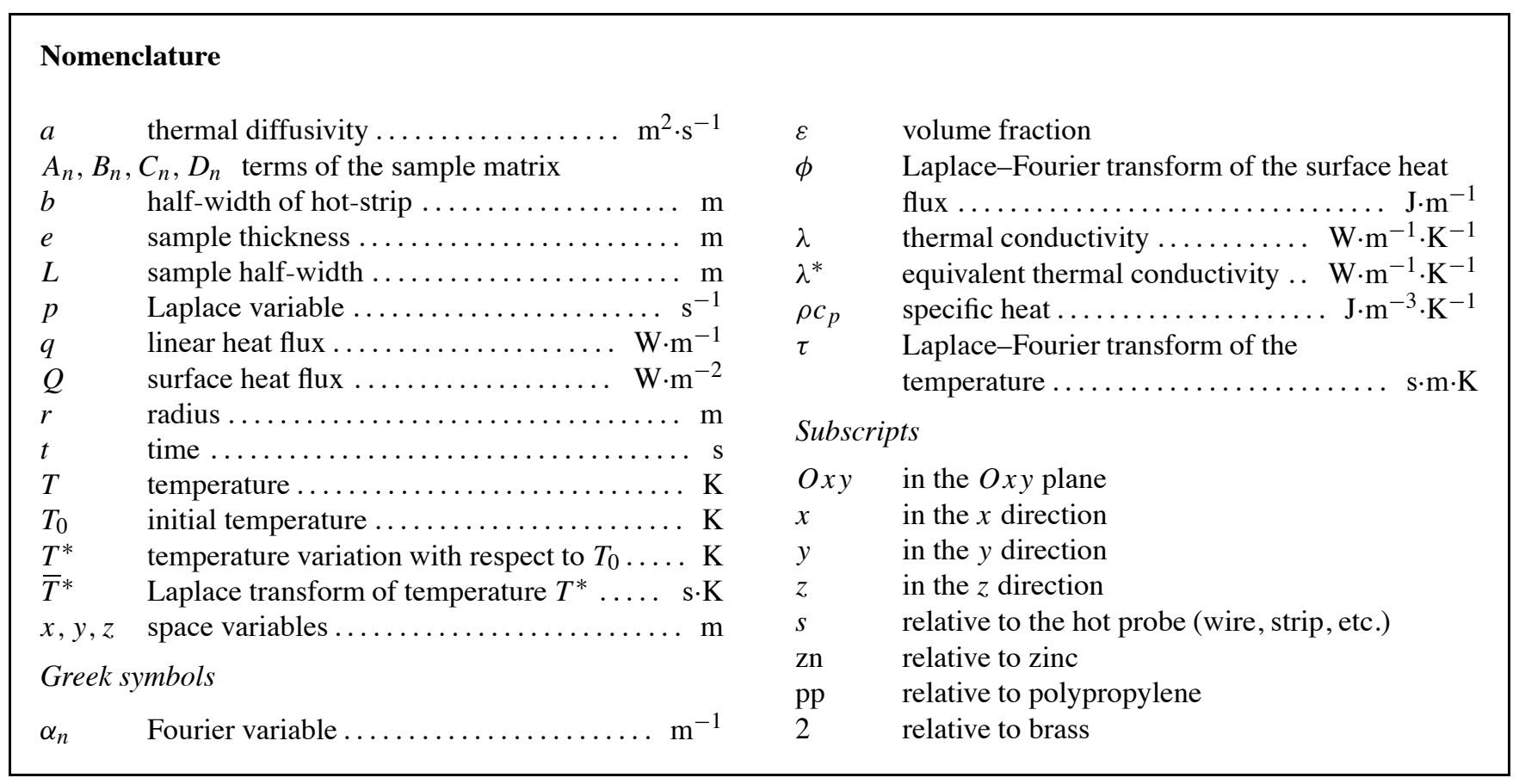

the wire axis is aligned with one of the main directions of the conductivity tensor. If the conductivity is isotropic in a plane perpendicular to the wire axis, the heat flux is radial and the measurement provides a thermal conductivity corresponding to the components denoted $\lambda_{x}$ and $\lambda_{y}$, where $\lambda_{x}=\lambda_{y}=\lambda_{O x y}$.

The hot-strip method is an extension of the hot-wire method wherein the heating element has a different design. The hot-wire is replaced by a hot-strip assimilated to a flat source whose lateral extension is small but whose length is assumed infinite.

The aim of the work described herein was to use both the hot-wire and hot-strip methods. The hot-wire measurement gives access to $\lambda_{O x y}$ and the hot-strip allows identification of the transverse conductivity $\lambda_{z}$ based on the other two components obtained by the hot-wire method.

After a brief review of the two methods and a description of the devices used, we propose a model of the hot-strip measurement on an orthotropic material, the associated identification method and the initial validation results on a well-known specimen material, the multilayer.

\section{Experimental methods}

\subsection{Hot-wire}

\subsubsection{Principle of the method}

The principle of the hot-wire-method is as follows: a resistant wire is placed on the axis of a very long cylindrical sample whose radial extension is assumed infinite. The sample, initially isothermal, is subjected to a pulsed heat flux produced as a Joule effect by the resistant wire. If the wire is assumed to have an infinite length and a negligible radius, heat transfer is radial and the variation of the temperature in a point located at a distance $r$ from the sample axis is expressed [16]:

$$
T(r, t)-T_{0}=\frac{q}{4 \pi \lambda} \int_{u=\frac{r^{2}}{4 a t}}^{+\infty} \frac{\mathrm{e}^{-u}}{u} \mathrm{~d} u
$$

where $q$ is the linear heat flux dissipated by the wire, $\lambda$ is the thermal conductivity of the material, $a$ is its thermal diffusivity and $T_{0}$ is the initial temperature of the medium. Asymptotic development of the above integral leads to an approximated solution with the form:

$$
T(r, t)-T_{0}=\frac{q}{4 \pi \lambda}\left[\ln \left(t^{*}\right)-C-\sum_{n=1}^{\infty}(-1)^{n} \frac{1}{t^{* n} n n !}\right]
$$

where $t^{*}=4 a t / r^{2}$ and $C=0.5772$ is the Euler constant.

For sufficiently long times and/or for low values of $r$ $\left(a t / r^{2} \gg 1\right)$, terms $1 / t^{* n}$ approach zero. This then yields a variation with time of the temperature which is linear in $\ln (t)$, i.e.:

$T(r, t)-T_{0}=\frac{q}{4 \pi \lambda}[\ln (t)+K]$

where $K=\ln \left(4 a / r^{2}\right)-C$.

If a thermocouple is placed in a fixed point $r$ to record the variation with time of the temperature, the slope of curve $T^{*}=T(r, t)-T_{0}=f(\ln (t))$ over long times allows identification of the thermal conductivity, where the linear flux $q$ produced by the resistant wire is assumed known.

When the resistant wire and thermocouple are one and the same, the temperature from which the thermal conductivity is identified is the surface temperature of the wire or the average wire temperature. 
Various papers [5-7] have shown that the inertia of the hot-wire has no influence on the identified conductivity provided the length of time is correctly chosen. As concerns the existence of possible resistance at the interface between hot-wire and sample, its influence affects the initial ordinate value but not the slope of $T\left(r_{s}, t\right)-T_{0}=f(\ln (t))$ [3, 17]. We will therefore preserve this simple identification procedure, considering that the time above which it is valid is determined directly from the experimental thermogram. For short times, the form of the thermogram is conditioned by hot-wire thermal inertia effects. Then, after a certain time, the temperature is observed to vary linearly as a function of $\ln (t)$. Identification is carried out in this second part of the thermogram.

\subsubsection{Experimental setup}

The hot-wire probe we used is sold by TELEPH ( 38240 Meylan, France) and is based on the work of Quénard and Sallée [18]. This probe is manufactured by a process similar to that used for printed circuit boards. It consists of a heating element (wire) and a thermocouple etched on a composite copper-polyimide-constantan film ensuring the mechanical strength of the system (Fig. 1). The heating element is connected to a stabilised power supply and the thermocouple signal is recorded after amplification on a digital oscilloscope. The heating element has an effective length of $5 \mathrm{~cm}$ and the probe thickness is approximately $4 / 10 \mathrm{~mm}$. The probe must be placed between two identical samples of sufficient size that they behave like a medium with an infinite radial dimension with respect to the wire.

\subsection{Hot-strip}

\subsubsection{Principle of the method}

The principle of the method is similar to the hot-wire method except for the difference in geometry of the probe, as shown in Fig. 2. The hot-strip probe is totally symmetrical. It consists of a rectangular heating element fitted with a thermocouple that measures the temperature variations of the probe itself. If the probe is placed in a medium with an infinite extension, this temperature variation can be expressed $[9,11]$ :

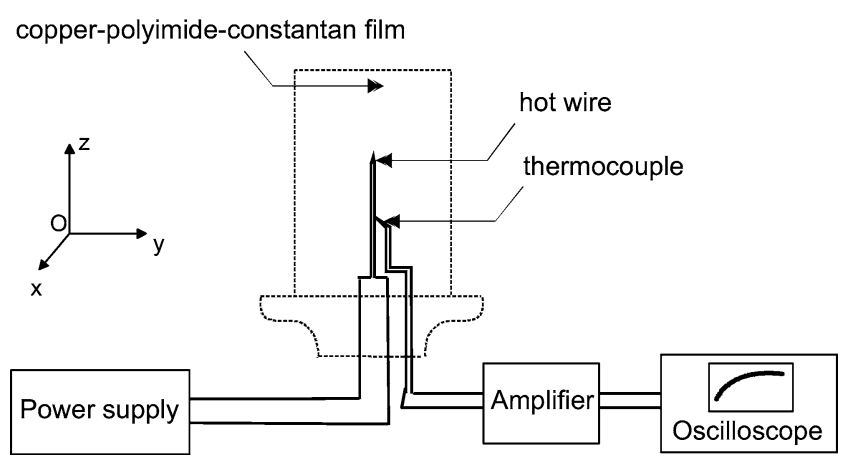

Fig. 1. Design of the hot-wire device.

$$
\begin{aligned}
& T(0, y, 0, t)-T_{0} \\
& =\frac{1}{4 \lambda \sqrt{\pi}} \int_{0}^{2 \sqrt{a t}} Q\left[\operatorname{erfc}\left(\frac{y-b}{u}\right)-\operatorname{erfc}\left(\frac{y+b}{u}\right)\right] \mathrm{d} u
\end{aligned}
$$

where $b$ is the half-width of the hot-strip and $Q$ the surface heat dissipation.

This solution corresponds to the case where longitudinal extension of the strip, $2 h$, is large compared with its width, $2 b$, allowing the transfers along $O x$ to be neglected. Furthermore, since the probe thickness is negligible (below a few tenths of a mm), it is considered that the temperature measurement is made in the neighbourhood of $z \approx 0$.

\subsubsection{Description of the manipulation}

In the context of a study designed to measure the conductivity of superinsulating isotropic materials, a device based on the hot-strip principle was adapted and developed at the Ecole des Mines of Albi (France) (Fig. 2). The probe $\left(2 h=80 \mathrm{~mm}, 2 b=3.2 \mathrm{~mm}, e_{s}=0.5 \mathrm{~mm}\right)$, similar in design to the hot-wire probe already described, was placed between two identical rectangular samples $(2 L=$ $45 \mathrm{~mm}, e \leqslant 20 \mathrm{~mm}$ and length $=100 \mathrm{~mm}$ ), themselves placed between two brass blocks (same size as the samples, thickness $=55 \mathrm{~mm}$ ). The strip temperature was measured by thermocouple (Fig. 2). A stabilised power supply provided a voltage across the heating element. The temperature rise was recorded by an acquisition card. The waveform and amplitude of the resulting signal were determined by the thermal conductivity and specific heat of the material.

A model taking the inertial effect of the hot-strip and the thermal resistance on the strip-sample interface into account was developed using integral transformations of the transfer equation and quadrupole formalism. It supplied an analytic solution of the hot-strip temperature variation [14]. The thermal values, i.e., the thermal conductivity and specific heat, were identified by an iterative minimisation method.

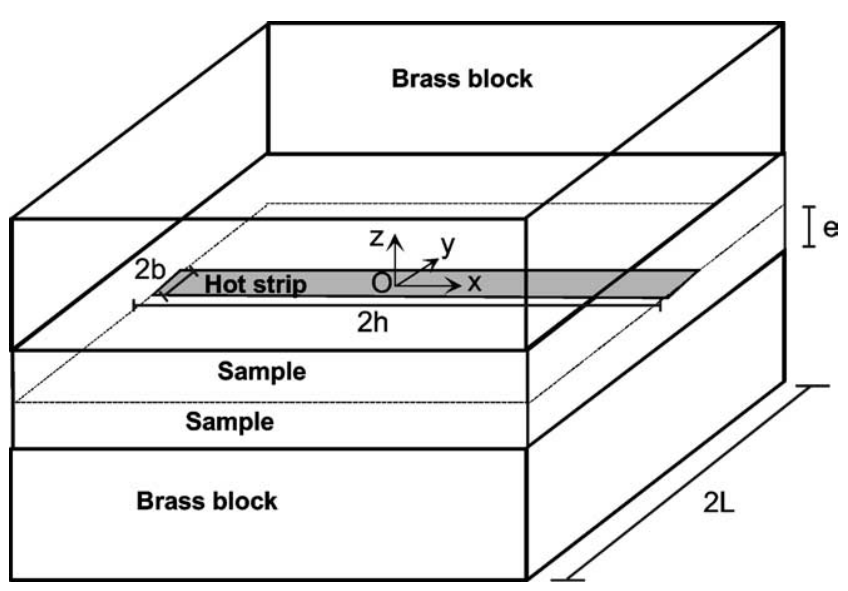

Fig. 2. Design of the hot-strip device for characterisation of insulating media. 
Our contribution consisted of using the same approach to model heat transfer in an orthotropic material in order to identify the transverse component of the conductivity tensor.

\section{Development of the procedure for measuring the transverse conductivity $\lambda_{z}$}

\subsection{Modelling of heat transfer in the material}

The symmetries of the problem led to modelling one quarter of the domain (Fig. 3). Considering the aspect ratio of the strip, its length was assumed infinite, allowing us to neglect thermal diffusion along the strip (along $O x$ ). This reduced the problem to be solved to a two-dimensional problem in plane $O y z$. The conductivity in the plane $z=0$ is denoted $\lambda_{O x y}$ and that in direction $O z$ is denoted $\lambda_{z}$

Assuming orthotropy, the $2 \mathrm{D}$ heat equation is written:

$$
\begin{aligned}
& \lambda_{O x y} \frac{\partial^{2}}{\partial y^{2}} T(y, z, t)+\lambda_{z} \frac{\partial^{2}}{\partial z^{2}} T(y, z, t) \\
& \quad=\rho c_{p} \frac{\partial}{\partial t} T(y, z, t)
\end{aligned}
$$

We used integral transformations to solve the problem analytically, i.e., establish the expression for the strip temperature to determine, by an appropriate identification method, the thermal parameters to be used to reset it on the experimental data. The heat equation was solved by applying a Laplace transform to variable $t$ then a Fourier cosine transform to variable $y$. The solution itself is then obtained by electrical analogy (quadrupole method [19]).

Below, we consider the temperature variation with respect to the initial time by setting $T^{*}=T-T_{0}$, where $T_{0}$ is the initial sample temperature, assumed uniform. In the Laplace space, the heat equation becomes:

$\lambda_{O x y} \frac{\partial^{2} \overline{T^{*}}}{\partial y^{2}}+\lambda_{z} \frac{\partial^{2} \overline{T^{*}}}{\partial z^{2}}-\rho c_{p} p \overline{T^{*}}=0$

where $\overline{T^{*}}(y, z, p)$ is the Laplace transform of $T^{*}(y, z, t), p$ being the Laplace variable.

Let $\tau\left(\alpha_{n}, z, p\right)$ be the Fourier cosine transform of $\overline{T^{*}}(y$, $z, p)$ :

$$
\begin{aligned}
\tau( & \left.\alpha_{n}, z, p\right) \\
& =\int_{0}^{L} T^{*}(y, z, p) \cos \left(\alpha_{n} y\right) \mathrm{d} y \\
& =\int_{0}^{+\infty} \int_{0}^{L} T(y, z, t) \mathrm{e}^{-p t} \cos \left(\alpha_{n} y\right) \mathrm{d} y \mathrm{~d} t
\end{aligned}
$$

where $\alpha_{n}=n \pi / L$.

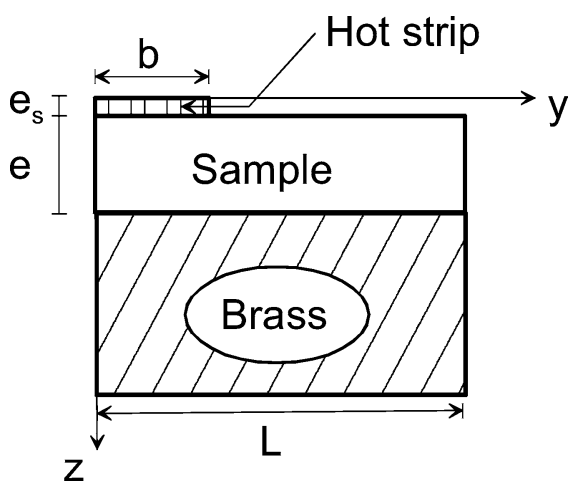

Fig. 3. Domain modelled.

This Fourier cosine transform is applied to the heat equation in the Laplace space to arrive at the transfer equation in the Laplace-Fourier space with the form:

$\frac{\mathrm{d}^{2}}{\mathrm{~d} z^{2}} \tau\left(\alpha_{n}, z, p\right)=k_{n}^{2} \tau\left(\alpha_{n}, z, p\right)$

where

$k_{n}=\sqrt{\frac{p}{a_{z}}+\frac{\lambda_{O x y}}{\lambda_{z}} \alpha_{n}^{2}}, \quad a_{z}=\frac{\lambda_{z}}{\rho c_{p}}$

The general solution of such an equation has the form:

$\tau\left(\alpha_{n}, z, p\right)=K_{1} \mathrm{e}^{k_{n} z}+K_{2} \mathrm{e}^{-k_{n} z}$

We used the electrical analogy to obtain the solution corresponding to our experimental context.

The entire device can be modelled as a sequence of quadrupoles to relate the temperature and heat flux on the temperature probe to the sample outlet temperature and heat flux [19]. Each medium can be characterised by a transfer matrix with dimension $(2 \times 2)$ relating the medium inlet temperature and heat flux to the medium outlet temperature and heat flux. The transfer matrices relative to each element are obtained from the general solutions of the transfer equation in the Fourier-Laplace space taking the specific role of each element into account.

Matrix associated with the material (passive medium):

$\left[\begin{array}{ll}A_{n} & B_{n} \\ C_{n} & D_{n}\end{array}\right]=\left[\begin{array}{cc}\cosh \left(k_{n} e\right) & \frac{\sinh \left(k_{n} e\right)}{\lambda_{z} k_{n}} \\ \lambda_{z} k_{n} \sinh \left(k_{n} e\right) & \cosh \left(k_{n} e\right)\end{array}\right]$

yielding:

$\left[\begin{array}{c}\tau\left(\alpha_{n}, e_{s}, p\right) \\ \phi\left(\alpha_{n}, e_{s}, p\right)\end{array}\right]=\left[\begin{array}{cc}A_{n} & B_{n} \\ C_{n} & D_{n}\end{array}\right]\left[\begin{array}{c}\tau\left(\alpha_{n}, e+e_{s}, p\right) \\ \phi\left(\alpha_{n}, e+e_{s}, p\right)\end{array}\right]$

Matrix associated with the probe (source term):

$\left[\begin{array}{ll}A_{s} & B_{s} \\ C_{s} & D_{s}\end{array}\right]=\left[\begin{array}{cc}1 & 0 \\ \left(\rho c_{p}\right)_{s} e_{s} p \frac{b}{L} & 1\end{array}\right]$

yielding:

$\left[\begin{array}{c}\tau\left(\alpha_{n}, 0, p\right) \\ \phi\left(\alpha_{n}, 0, p\right)\end{array}\right]=\left[\begin{array}{ll}A_{s} & B_{s} \\ C_{s} & D_{s}\end{array}\right]\left[\begin{array}{c}\tau\left(\alpha_{n}, e_{s}, p\right) \\ \phi\left(\alpha_{n}, e_{s}, p\right)\end{array}\right]$ 
The brass block, a very good heat conductor, is designed to create a heat sink to absorb the heat dissipated by the probe and thereby preferentially orient the heat flux toward the brass masses instead of the sides of the sample. It is also used to homogenise and control the temperature at the rear. Furthermore, the brass mass can be considered as a semiinfinite medium. These observations are expressed by the relation:

$\phi\left(\alpha_{n}, e_{s}+e, p\right)=\lambda_{2} \sqrt{\frac{p}{a_{2}}+\alpha_{n}^{2}} \tau\left(\alpha_{n}, e_{s}+e, p\right)$

where $\lambda_{2}$ and $a_{2}$ are the thermal conductivity and diffusivity respectively of the brass.

This gives a matrix equation relating the temperature and heat flux on the temperature probe to the temperature and heat flux at the rear of the sample with the form:

$$
\begin{aligned}
& {\left[\begin{array}{c}
\tau\left(\alpha_{n}, 0, p\right) \\
\phi\left(\alpha_{n}, 0, p\right)
\end{array}\right]} \\
& \quad=\left[\begin{array}{cc}
1 & 0 \\
\left(\rho c_{p}\right)_{s} e_{s} p \frac{b}{L} & 1
\end{array}\right]\left[\begin{array}{cc}
A_{n} & B_{n} \\
C_{n} & D_{n}
\end{array}\right] \\
& \quad \times\left[\begin{array}{c}
\left.\alpha_{n}, e+e_{s}, p\right) \\
\phi\left(\alpha_{n}, e+e_{s}, p\right)=\lambda_{2} \sqrt{\alpha_{n}^{2}+\frac{p}{a_{2}}} \tau\left(\alpha_{n}, e+e_{s}, p\right)
\end{array}\right]
\end{aligned}
$$

In this equation, the thermal contact resistance between the hot-strip and sample and between the sample and brass block were neglected. This approximation is valid insofar as the thermal resistance of the sample is much greater than $10^{-4} \mathrm{~m}^{2} \cdot \mathrm{K} \cdot \mathrm{W}^{-1}$ [14]. This constraint thus defines the domain of validity of the procedure proposed herein.

\subsection{Obtaining of a model thermogram}

\subsubsection{General behaviour}

In the Laplace-Fourier space, modelling by the quadrupole method gives the expression of the temperature over time in the location where it is measured by the thermocouple, i.e., in $z=0$.

$$
\begin{aligned}
\tau\left(\alpha_{n}, 0, p\right) \\
=\left\{A_{n}+D_{n} \lambda_{2} \sqrt{\frac{p}{a_{2}}+\alpha_{n}^{2}}\right\} \phi\left(\alpha_{n}, 0, p\right) \\
\quad \times\left\{C_{n}+A_{n}\left(\rho c_{p}\right)_{s} e_{s} p \frac{b}{L}\right. \\
\left.\quad+\lambda_{2} \sqrt{\frac{p}{a_{2}}+\alpha_{n}^{2}}\left[B_{n}\left(\rho c_{p}\right)_{s} e_{s} p \frac{b}{L}+D_{n}\right]\right\}^{-1}
\end{aligned}
$$

The expression for $\phi\left(\alpha_{n}, 0, p\right)$ is obtained by applying a Laplace-Fourier transform to the thermal flux density $Q$ produced by the hot-strip in $z=0$, i.e.:

$\phi\left(\alpha_{n}, 0, p\right)=\frac{Q}{p} \frac{\sin \left(\alpha_{n} b\right)}{\alpha_{n}}$

The temperature variation in the real space is then determined during a first stage from an inverse Fourier transform with the form:

$$
\begin{aligned}
\overline{T^{*}}(y, 0, p)= & \frac{1}{L} \tau(0,0, p) \\
& +\frac{2}{L} \sum_{n=1}^{\infty} \tau\left(\alpha_{n}, 0, p\right) \cos \left(\alpha_{n}, y\right)
\end{aligned}
$$

Considering that the temperature is measured at $y=0$, we were particularly interested in:

$\overline{T^{*}}(0,0, p)=\frac{1}{L} \tau(0,0, p)+\frac{2}{L} \sum_{n=1}^{\infty} \tau\left(\alpha_{n}, 0, p\right)$

The inverse Laplace transform recommended by [14] is calculated numerically and leads to the model thermogram:

$T^{*}(0,0, t)=T(0,0, t)-T_{0}$

As can be seen from Eqs. (9), (11), (17) and (20), the temperature variation on the probe depends of course on the thermal characteristics of the sample $\left(\lambda_{O x y}, \lambda_{z}\right.$ and $\left.\rho c_{p}\right)$ but also on the thermal characteristics of the hot-strip and the brass blocks. The data for brass are widely available in the literature. For the hot wire, a measurement was made with reference samples to identify its specific heat once and for all. Once all the invariant parameters have been determined, only two quantities remain to be identified: the specific heat of the sample, $\rho c_{p}$, and its transverse conductivity, $\lambda_{z}$.

\subsubsection{Asymptotic behaviour over long times}

After the initial moments, the capacitive effect of the hotstrip is no longer detectable on the thermogram $\left(\left(\rho c_{p}\right)_{s} \approx\right.$ $0)$. Similarly, for a sufficiently long time, the capacitive effect of the sample can be neglected $\left(\rho c_{p} \approx 0\right)$. Furthermore, the conductivity of the brass is very high compared with the insulating media for which the device is adapted, i.e., $\lambda_{2} \gg \lambda_{z}$. Considering all these remarks, applying the same approximations as for isotropic material [14], the general solution (Eq. (17)) can be simplified to the following form:

$$
\begin{aligned}
\tau\left(\alpha_{n}, 0, p\right)= & \frac{1}{\lambda_{2} \sqrt{\frac{p}{a_{2}}+\alpha_{n}^{2}} \frac{Q}{p} \frac{\sin \left(\alpha_{n} b\right)}{\alpha_{n}}} \\
& +\frac{\tanh \left(\sqrt{\frac{\lambda_{O x y} \alpha_{n}^{2}}{\lambda_{z}}} e\right)}{\lambda_{z} \sqrt{\frac{\lambda_{O x y} \alpha_{n}^{2}}{\lambda_{z}}}} \frac{Q}{p} \frac{\sin \left(\alpha_{n} b\right)}{\alpha_{n}}
\end{aligned}
$$

which, after inverse transformation to return to the real space, yields in $y=0$ :

$$
\begin{aligned}
T^{*}(0,0, p)= & \frac{Q b}{L \sqrt{\pi \lambda_{2}\left(\rho c_{p}\right)_{2}}} \sqrt{t}+\frac{e Q b}{\lambda_{z} L} \\
& +\sum_{n=1}^{\infty} \frac{2 Q}{\lambda_{z} L} \frac{\tanh \left(\sqrt{\frac{\lambda_{O x y}}{\lambda_{z}}} \alpha_{n} e\right)}{\sqrt{\frac{\lambda_{\text {Oxy }}}{\lambda_{z}}} \alpha_{n}} \frac{\sin \left(\alpha_{n} b\right)}{\alpha_{n}}
\end{aligned}
$$

This analysis of the system's behaviour over a long time shows that the thermogram approaches a straight line in $\sqrt{t}$ with an initial ordinate value for which the only unknown is $\lambda_{z}$. This result is used to initiate the identification procedure described in the next section. 

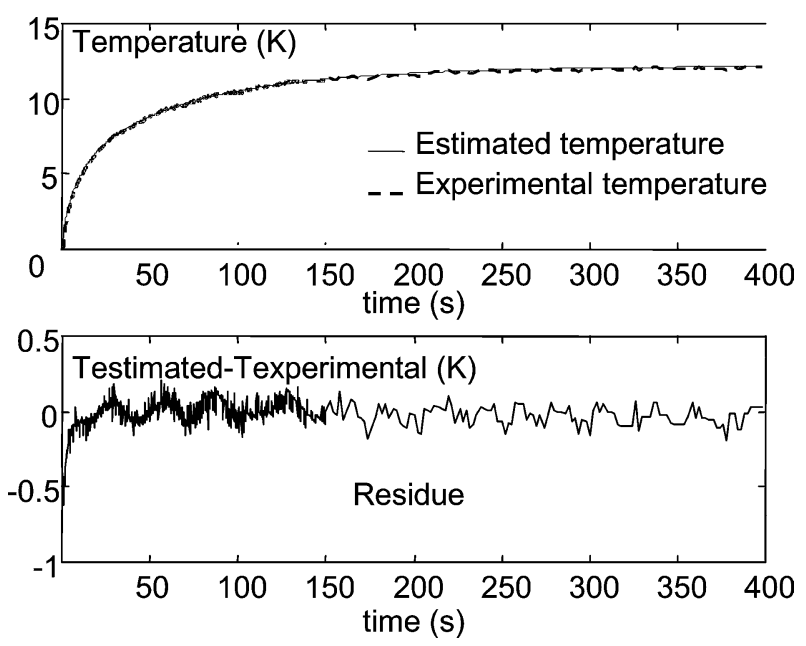

Fig. 4. Experimental and estimated thermograms: Represention of residues after minimization.

\subsection{Material transverse conductivity identification procedure}

The model parameter sensitivity analysis made by [14] for the case of an isotropic material shows that the thermal conductivity and specific heat of the sample are effectively identifiable, with greater sensitivity for the thermal conductivity. Since our aim is to measure the transverse conductivity $\lambda_{z}$ of the material, only the results concerning this parameter are presented.

Identification is carried out by a conventional method for minimising the differences between the experimental thermogram and the theoretical thermogram determined from Eq. (21) (Nelder-Mead's minimisation algorithm [20]). Since the complete problem is not linear in the sense of the parameters (Eqs. (17)-(21)), direct inversion is not possible. The parameters are estimated by an iterative process. The iterative process is initiated using the conductivity determined from the initial ordinate value of the constitutive equation over long times (Eq. (23)). The quality of identification can be illustrated by representing the residue of the estimate (difference between experimental and calculated thermograms) due to measurement noise and the parameter estimation error. An example corresponding to the experimental results described in the next section is given in Fig. 4.

The bias observed for short periods is related to the fact that the model is less sensitive to specific heat, whose influence predominates at first, than to thermal conductivity. This bias does not disappear completely even when a much larger number of experimental points are taken into account over short periods.

\section{Experimental validation and applications}

\subsection{Validation method}

To validate our approach, measurements were made on a two-phase laminated material for which it was possible to accurately determine the components of the thermal conductivity tensor. The measurement of the transverse conductivity across the strata knowing the conductivity parallel to the strata was compared with the expected theoretical value and allowed us to validate our approach.

In a multilayer, the contrast between the transverse and parallel thermal conductivity values is related to the intrinsic conductivities of the materials of the two phases and their volume fraction. To obtain correctly differentiated values, we designed a laminate consisting of a good conductor (zinc) and an insulator (polypropylene). Conventional measurements were made to obtain the thermal conductivity of the zinc $\left(\lambda_{\mathrm{zn}}=116 \mathrm{~W} \cdot \mathrm{m}^{-1} \cdot \mathrm{K}^{-1}\right)$ and polypropylene $\left(\lambda_{\mathrm{pp}}=0.17 \mathrm{~W} \cdot \mathrm{m}^{-1} \cdot \mathrm{K}^{-1}\right)$. The thickness of the elementary two-layer cell of the multilayer was $e=1.73 \mathrm{~mm}$ with a thickness $e_{\mathrm{pp}}=1.08 \mathrm{~mm}$ for the polypropylene and $e_{\mathrm{zn}}=$ $0.65 \mathrm{~mm}$ for the zinc, corresponding to volume fractions $\varepsilon_{\mathrm{pp}}=0.624$ and $\varepsilon_{\mathrm{zn}}=0.376$, respectively.

Conventionally, the thermal conductivity in the plane of the strata is expressed:

$\lambda_{O x y}=\varepsilon_{\mathrm{pp}} \lambda_{\mathrm{pp}}+\varepsilon_{\mathrm{zn}} \lambda_{\mathrm{zn}}$

and the transverse thermal conductivity by:

$\lambda_{z}=\left(\frac{\varepsilon_{\mathrm{zn}}}{\lambda_{\mathrm{zn}}}+\frac{\varepsilon_{\mathrm{pp}}}{\lambda_{\mathrm{pp}}}\right)^{-1}$

To validate the orthotropic model, the thermal conductivity $\lambda_{z}$ of the laminated medium was measured, including in the data the thermal conductivity parallel to the strata $\lambda_{O x y}$ calculated before. Considering the thermophysical properties of zinc and polypropylene, this yields $\lambda_{O x y}=$ $43.7 \mathrm{~W} \cdot \mathrm{m}^{-1} \cdot \mathrm{K}^{-1}$. The expected value of the transverse conductivity was $\lambda_{z}=0.27 \mathrm{~W} \cdot \mathrm{m}^{-1} \cdot \mathrm{K}^{-1}$.

\subsection{Validation measurement results}

Measurements were made on several samples of different thicknesses (variable number of elementary cells) varying the heat flux produced by the hot-strip. Table 1 gives the identification results obtained.

Although a dispersion in the measurements is observed (from $-7 \%$ to $6 \%$ ), the very small difference between the average value of all these measurements $\left(\bar{\lambda}_{z}=0.275\right.$ $\left.\mathrm{W} \cdot \mathrm{m}^{-1} \cdot \mathrm{K}^{-1}\right)$ and the expected value of the equivalent transverse thermal conductivity $\left(\lambda_{z}=0.272 \mathrm{~W} \cdot \mathrm{m}^{-1} \cdot \mathrm{K}^{-1}\right)$ demonstrates the reliability of the hot-strip measurement method under the assumption of orthotropy.

If we consider the case of isotropic material [14], the validity and identifiability domain of the method was defined by a systematic study of the model's sensitivity to various parameters such as the lateral convective losses of the sample, the thermal conductivity of the conductive blocks, the thermal and dimensional characteristics of the probe and the heat dissipated in the sample. The method is validated if the thermal conductivity of the sample is less than $0.5 \mathrm{~W} \cdot \mathrm{m}^{-1} \cdot \mathrm{K}^{-1}$ and its specific heat is between 
Table 1

Thermal conductivity measurements of a multilayer medium

\begin{tabular}{lllll}
\hline & $\begin{array}{l}\text { Measurement 1 } \\
(5 \text { cells })\end{array}$ & $\begin{array}{l}\text { Measurement 2 } \\
(5 \text { cells })\end{array}$ & $\begin{array}{l}\text { Measurement 4 } \\
(3 \text { cells })\end{array}$ & $\begin{array}{l}\text { Measurement 5 } \\
(2 \text { cells })\end{array}$ \\
\hline Sample thickness [mm] & 8.65 & 8.65 & 5.19 & 3.46 \\
Voltage across the strip [V] & 5.5 & 6 & 7 & 7.5 \\
$\begin{array}{l}\text { Measured transverse conductivity } \lambda_{z} \\
{\left[\mathrm{~W} \cdot \mathrm{m}^{-1} \cdot \mathrm{K}^{-1} \text { ] }\right.}\end{array}$ & 0.253 & 0.288 & 0.274 & 0.284 \\
$\begin{array}{l}\text { Relative difference between measured } \\
\text { and estimated values of } \lambda_{z}\end{array}$ & $-7.0 \%$ & $+5.9 \%$ & $+0.7 \%$ & $+4.4 \%$ \\
\hline
\end{tabular}

Table 2

Comparison between the estimated thermal conductivity of an isotropic model and an orthotropic model

\begin{tabular}{llll}
\hline & $\begin{array}{l}\lambda_{\text {Oxy }} \text { measured by } \\
\text { hot-wire } \\
{\left[\mathrm{W} \cdot \mathrm{m}^{-1} \cdot \mathrm{K}^{-1}\right]}\end{array}$ & $\begin{array}{l}\lambda_{z} \text { measured by hot-wire } \\
(\text { isotropic model) } \\
{\left[\mathrm{W} \cdot \mathrm{m}^{-1} \cdot \mathrm{K}^{-1}\right]}\end{array}$ & $\begin{array}{l}\lambda_{z} \text { measured by hot-strip } \\
(\text { orthotropic model) } \\
{\left[\mathrm{W} \cdot \mathrm{m}^{-1} \cdot \mathrm{K}^{-1}\right]}\end{array}$ \\
\hline Sample 1 & 0.112 & 0.080 & 0.058 \\
Sample 2 & 0.109 & 0.075 & 0.050 \\
Sample 3 & 0.097 & 0.075 & 0.058 \\
Sample 4 & 0.103 & 0.075 & 0.056 \\
\hline
\end{tabular}

$5 \times 10^{4} \mathrm{~J} \cdot \mathrm{m}^{-3} \cdot \mathrm{K}^{-1}$ and $10^{6} \mathrm{~J} \cdot \mathrm{m}^{-3} \cdot \mathrm{K}^{-1}$. In the example discussed herein, the anisotropy was very pronounced and the conductivity in the plane of the strata was very high $\left(\lambda_{\text {Oxy }}=43.7 \mathrm{~W} \cdot \mathrm{m}^{-1} \cdot \mathrm{K}^{-1}\right)$. This gives reason to believe that the dispersion in the measurements observed is probably related to a large lateral flux leading to non-negligible convective losses in the case where the material is very thick, since the largest differences are observed for a laminate with a thickness of $8.65 \mathrm{~mm}$ (Table 1).

For the new application of this measuring device to anisotropic media, it therefore appears necessary to plan a complete sensitivity analysis to detail the constraints to be satisfied for the two conductivity values, $\lambda_{O x y}$ and $\lambda_{z}$. This being said, the method described herein can already be used advantageously to characterise orthotropic media.

The estimation error can be evaluated using a linear model in the sense of the parameters over long times, by calculating the covariance matrix [14]:

$\operatorname{cov}\left[e_{\beta}\right]=\left([\mathbf{X}]^{\mathrm{T}}[\mathbf{X}]\right)^{-1} \sigma^{2}$

where $e_{\beta}$ is the estimation error on parameter $\beta, \mathbf{X}$ is the sensitivity matrix and $\sigma$ is the standard deviation related to the measurement noise. For the measurements made, the error on the initial ordinate value is around $0.1 \%$ for a standard deviation of $10 \%$.

\subsection{Thermal conductivity measurements on fibrous materials}

The method proposed was used to characterise nonwoven fibrous materials obtained by pneumatic layering, which are isotropic in the plane of the layers because of the random distribution of the fibres in each layer. The measurements made by the hot-wire method, with the hot-wire located perpendicular to the layers, give the value of $\lambda_{O x y}$. Other measurements made by hot-wire, placing the wire parallel to the layers give an apparent thermal conductivity $\lambda_{z}^{*}$ (isotropic model). These values are compared with those obtained by the hot-strip method (Table 2). These results show that neglecting the orthotropic nature of the material would lead to substantially overestimating the transverse conductivity, by as much as $50 \%$ in the case of the material considered, showing the interest of the work presented.

\section{Conclusion}

The initial results given in this paper are encouraging, since they show how, using an existing manipulation and without modifying the device, it is possible, with a different model, to identify the thermal conductivities of an orthotropic material exhibiting transverse isotropy.

Certain points should be stressed. In work relative to the hot-wire, certain problems may arise because of the heterogeneous nature of the materials to be characterised. Since the sample is assumed to be infinite, although the observation is made on a macroscopic scale far larger than the scale of the representative volume element, the scales related to the observation time remain small, even if they are long compared with the wire inertia. If the conductivity contrasts are very large, on the wire-sample interface the transfers are very different at the contact with each of the two phases, and the description of the transfer on the macroscopic scale by a conventional model is not possible [21]. Since the hot-wire measurement is a given in our problem of transverse conductivity identification, any error in this value is reflected in the characterisation method. It is therefore necessary to be very cautious if the contrasts 
between phases are very large, which is the case for our test material $\left(\lambda_{\mathrm{zn}} / \lambda_{\mathrm{pp}}=682\right)$. We therefore measured the influence of an error in the determination of $\lambda_{O x y}$ on the identified value of $\lambda_{z}$. In this specific case, an error of $\pm 15 \%$ on the value of $\lambda_{\text {Oxy }}$ leads to an error not exceeding $\pm 3 \%$ on the identified value of the transverse conductivity $\lambda_{z}$ which is entirely acceptable.

Furthermore, it should be noted that the model developed can be applied to any orthotropic materials such that $\lambda_{x} \neq$ $\lambda_{y} \neq \lambda_{z}$. In effect, since the hot-strip can be assimilated to an infinite strip because of the length/width ratio, heat transfer in the sample is two-dimensional. In this case, the remaining problem is to measure the conductivity $\lambda_{x}$ if it is different from $\lambda_{y}$.

\section{References}

[1] M. Amazouz, C. Moyne, A. Degiovanni, Measurement of the thermal diffusivity of anisotropic materials, High Temperatures-High Pressures 19 (1987) 37-41.

[2] I. Philippi, J.C. Batsale, D. Maillet, A. Degiovanni, Traitement d'images infrarouges par transformation intégrale-Application à la mesure de diffusivité thermique de matériaux anisotropes par méthode flash, Rev. Gén. Thermique 392-393 (1994) 486-496.

[3] P. Andersson, Thermal conductivity of some rubbers under pressure by the transient hot-wire method, J. Appl. Phys. 47 (6) (1976) 2424-2426.

[4] J.R. Bloomer, J. Ward, A semi-automatic field apparatus for the measurement of thermal conductivities of sedimentary rocks, J. Phys. E 12 (1979) 1033-1035.

[5] Y. Nagasaka, A. Nagashima, Simultaneous measurement of the thermal conductivity and the thermal diffusivity of liquids by the transient hot-wire method, Rev. Sci. Instrum. 52 (2) (1981) 229-232.

[6] G. Backstrom, Determination of thermal physical properties using a shielded thermocouple, J. Phys. E 15 (1982) 1049-1053.

[7] R.A. Perkins, R. McAllister, E.D. Sloan, M.S. Graboski, A new transient hot-wire thermal conductivity instrument for use with both step power and ramp power forcing, Thermal Conductivity 18 (1984) 273-282.

[8] H. Lobo, C. Cohen, Measurement of thermal conductivity of polymer melts by the line source method, Poly. Engng. Sci. 30 (2) (1990) 6570 .
[9] S.E. Gustafsson, E. Karawacki, M.N. Khan, Transient hot-strip method for simultaneously measuring thermal conductivity and thermal diffusivity of solids and fluids, J. Phys. D: Appl. Phys. 12 (1979) 1411-1421.

[10] S.E. Gustafsson, E. Karawacki, M.A. Chohan, Thermal transport studies of electrically conducting materials using the transient hot-strip technique, J. Phys. D: Appl. Phys. 19 (1986) 727-735.

[11] R. Singh, N.S. Saxena, D.R. Chaudhary, Simultaneous measurement of thermal conductivity and thermal diffusivity of some building materials using the transient hot-strip method, J. Phys. D 18 (1985) $1-8$.

[12] T. Log, Transient hot-strip method for simultaneous determination of thermal conductivity and thermal diffusivity of refractory materials, J. Amer. Ceram. Soc. 74 (3) (1991) 650-653.

[13] T. Log, M.M. Metallinou, Thermal conductivity measurements using a short transient hot-strip method, Rev. Sci. Instrum. 63 (8) (1992) 3966-3971.

[14] B. Ladevie, J.C. Batsale, O. Fudym, Some methods to estimate the thermal conductivity of insulating media-Application to the characterization of fluids in laminar couette flow, in: Inverse Problems in Engineering: Theory and Practice Proceedings of 3rd International Conference on Inverse Problems in Engineering, Port Ludlow, USA, 1999.

[15] E. Coment, O. Fudym, J.C. Batsale, B. Ladevie, R. Santander, Extension of the hot-wire method to the characterisation of stratified soils with multiple temperature analysis, in: 4th Int. Conf. Inverse Problems in Engineering: Theory and Practice, Icipe 2002, Angra dos Reis, Brazil, May 26-31, 2002.

[16] H.S. Carslaw, J.C. Jaeger, Conduction of Heat in Solids, Oxford University Press, Oxford, 1959.

[17] J. Nicolas, P. André, J.F. Rivez, V. Debbaut, Thermal conductivity measurements in soil using an instrument based on the cylindrical probe method, Rev. Sci. Instrum. 64 (3) (1993) 774-780.

[18] D. Quénard, H. Sallée, Détermination rapide des paramétres thermiques des matériaux par sonde à choc et thermofluxmètres, Cahiers du CSTB 2295 (1988) 1-12.

[19] D. Maillet, S. André, J.C. Batsale, A. Degiovanni, C. Moyne, Thermal Quadrupoles, Solving the Heat Equation Through Integral Transforms, Wiley, New York, 2000.

[20] J.A. Nelder, R. Mead, A simplex method for function minimization, Comput. J. 7 (1965) 308-313.

[21] G.C. Glaztmaier, W.F. Ramirez, Use of volume averaging for the modeling of thermal properties of porous materials, Chem. Engng. Sci. 43 (12) (1988) 3157-3169. 